\title{
An Early Phase of Embryonic DIx5 Expression Defines the Rostral Boundary of the Neural Plate
}

\author{
Lu Yang, ${ }^{1,2}$ Hailan Zhang, ${ }^{1,3}$ Gezhi Hu, ${ }^{1,3}$ Hongyu Wang, ${ }^{1,2}$ Cory Abate-Shen, ${ }^{1,3}$ and Michael M. Shen ${ }^{1,2}$ \\ ${ }^{1}$ Center for Advanced Biotechnology and Medicine, and Departments of ${ }^{2}$ Pediatrics and ${ }^{3}$ Neuroscience and Cell Biology, \\ University of Medicine and Dentistry of New Jersey-Robert Wood Johnson Medical School, Piscataway, \\ New Jersey 08854
}

\begin{abstract}
Relatively little is known about the molecular events that specify the rostrocaudal axis of the neural plate. Here we show that a member of the Distal-less $(D / x)$ homeobox gene family, $D / x 5$, is one of the earliest known markers for the most rostral ectoderm, before the formation of an overt neural plate. During late gastrulation $D / \times 5$ expression becomes localized to the anterior neural ridge, which defines the rostral boundary of the neural plate, and also extends caudolaterally, marking the region of the presumptive neural crest. Subsequently, D/x5 is expressed in tissues (olfactory epithelium, ventral cephalic epithelium) that are believed to derive from the anterior neural ridge, based on the avian fate map. The early phase of D/x5 expression in the anterior neural ridge and its derivatives is distinct from a later phase of expression in the ventral telencephalon and dienceph-
\end{abstract}

During gastrulation stages of vertebrate embryogenesis the rostrocaudal axis of the neuroectoderm is specified via planar and vertical signaling events (for review, see Wilson and HemmatiBrivanlou, 1997). Several studies indicate that the subsequent patterning of the rostral neuroectoderm is mediated by the activities of local organizing centers that confer regional identity within the forebrain and midbrain. In the mouse embryo it has been demonstrated that the anterior neural ridge, which is located at the rostral boundary of the neural plate, produces organizing activities that pattern the adjacent rostral prosencephalon at early postgastrulation stages (Shimamura and Rubenstein, 1997). Furthermore, it recently has been shown in the zebrafish embryo that forebrain organizing activities exist in the anterior neural boundary during gastrulation stages (Houart et al., 1998). These early patterning events presumably are dependent on the restricted expression of regulatory genes during gastrulation and neurulation (Puelles and Rubenstein, 1993; Rubenstein et al., 1994; Shimamura et al., 1995). Thus, the identification of regulatory genes that have specific boundaries of expression in the rostral ectoderm at the onset of neural plate formation should facilitate our understanding of neural patterning.

\footnotetext{
Received Feb. 26, 1998; revised July 22, 1998; accepted July 27, 1998.

This work was supported by funding from National Institutes of Health (C.A.-S. and M.M.S.), the National Science Foundation (M.M.S.), and the American Heart Association (H.Z. and G.H.). We thank Ira Black, Cheryl Dreyfus, Richard Nowakowski, John Pintar, and Mengqing Xiang for helpful discussions and comments on this manuscript.

L.Y., H.Z., and G.H. contributed equally to this work.

Correspondence should be addressed to Dr. Michael Shen, Center for Advanced Biotechnology and Medicine, 679 Hoes Lane, Piscataway, NJ 08854.

Copyright (C) 1998 Society for Neuroscience $0270-6474 / 98 / 188322-09 \$ 05.00 / 0$
}

alon and also appears to be unique for D/x5 among members of the $D / x$ family. Another distinctive feature of $D / x 5$ expression is the occurrence of an alternative transcript $(\delta D / x 5)$, which encodes a truncated protein lacking the homeodomain, and represents a significant fraction of total D/x5 transcripts at all embryonic stages that were examined. In contrast with fulllength DLX5, the $\delta \mathrm{DLX} 5$ truncated protein is deficient in DNAbinding activity and does not interact with the homeoprotein partner MSX1. Taken together, our findings suggest that DIx5 activity may be regulated via the expression of an alternative transcript and demonstrate that DIx5 marks the anterior boundary of the neural plate.

Key words: homeobox gene; transcription factor; alternative transcripts; anterior neural ridge; neural crest; forebrain

Members of the Distal-less $(D l x)$ gene family previously have been implicated in processes of neural patterning, particularly during later stages of forebrain development (Qiu et al., 1995; Anderson et al., 1997a,b). The Dlx genes comprise a highly conserved family of vertebrate homeobox genes that, like other members of the homeobox superfamily, are thought to act as regulatory molecules via the actions of their protein products as transcription factors. In the mouse, seven $D l x$ genes have been identified, with numerous homologs found in Amphioxus, zebrafish, newt, Xenopus, chick, rat, and human [compiled by Stock et al. (1996)]. Members of the Dlx family share a conserved homeodomain that mediates sequence-specific DNA binding as well as interactions with other homeoproteins, such as MSX1 (Zhang et al., 1997). Other conserved regions in DLX proteins are important for mediating their actions as transcriptional activators ((Zhang et al., 1997; H. Zhang, G. Hu, and C. Abate-Shen, unpublished data). Furthermore, the similarity among the murine $D l x$ genes extends to their expression patterns during development, because several members of this family (Dlx1, Dlx2, Dlx3, $D l x 5$, and $D l x 6)$ are expressed in overlapping spatial domains during forebrain development (Porteus et al., 1991, 1994; Price et al., 1991; Robinson et al., 1991; Bulfone et al., 1993a,b; Simeone et al., 1994).

In this report we present evidence indicating that one member of the murine $D l x$ family, $D l x 5$, is regulated via alternative mRNA processing and that it is expressed at the boundaries of the rostral neural plate. First, we show that Dlx5 is represented by at least two alternative transcripts, a full-length transcript that encodes an intact and biochemically active protein and a second transcript that is lacking a homeodomain $(\delta D l \times 5)$. Second, we demonstrate that an early phase of $D l \times 5$ expression specifically 
defines the anterior boundary of the neural plate and, subsequently, the anterior neural ridge.

\section{MATERIALS AND METHODS}

Isolation of Dlx5 cDNA clones and biochemical analysis of DLX 5 protein products. We isolated a region of the Dlx 5 cDNA by reverse-transcriptase PCR (RT-PCR), using oligonucleotide primers that correspond to the 5' and $3^{\prime}$ ends of the coding sequence of the rat ortholog (Shirasawa et al., 1994). The sequences of these primers are $5^{\prime}$-GAG TCT GGA TCC ATG ACA GGA GTC TTT GAC AGA AGA-3' and 5' -CAT GTC GAA TTC AAG CTT CTA ATA AAG CGT CCC GGA GGC-3'. The resulting PCR product was cloned and used to screen a $\lambda$ Exlox (Stratagene, La Jolla, CA) cDNA library that we prepared from day 12.5 embryonic head mRNA. Five independent cDNA clones were isolated and sequenced. Four of these cDNA isolates are overlapping and $\sim 1.4 \mathrm{~kb}$ in size, with the longest isolate being identical in sequence to the published murine Dlx5 cDNA (Stock et al., 1996), which we confirmed was full-length by Northern blot analysis (data not shown). The fifth cDNA isolate, which we term $\delta D l x 5$, has an 82 bp internal deletion of nucleotides 514-595 [numbering is based on the cDNA sequence of Stock et al. (1996)] but is otherwise identical to the other Dl 55 cDNAs. The GenBank accession number for the full-length Dlx5 cDNA clone is $\mathrm{AF} 072452$ and for the $\delta$ Dlx 5 variant is AF072453.

To produce the DLX5 and $\delta$ DLX5 proteins, we cloned the inserts from the full-length and the $\delta$ Dlx 5 cDNA isolates into pGEM11zf (Promega, Madison, WI). RNA was prepared in vitro by using T7 RNA polymerase, followed by translation with rabbit reticulocyte lysates (Promega). To examine DNA binding activity, we performed gel mobility shift analysis by using in vitro translated proteins as described (Zhang et al., 1997). The sequences of the DNA sites that were used are shown in Figure $3 A$ and have been described (Catron et al., 1993). To examine protein-protein binding, we performed glutathione $S$-transferase (GST) interaction assays by using ${ }^{35} \mathrm{~S}$-labeled DLX5 or $\delta$ DLX 5 protein and GST-agarose or a GST-MSX1 fusion protein as described (Zhang et al., 1997).

Analysis of Dlx5 and $\delta$ Dlx5 expression. To examine Dlx5 expression, we dissected tissues or intact mouse embryos at the indicated developmental stage [in which $0.5 \mathrm{~d}$ post coitum (dpc) is defined as the morning of the copulatory plug] and prepared RNA by using Trizol reagent (Life Technologies, Gaithersburg, MD). Ribonuclease protection analysis was performed essentially as described (Shen and Leder, 1992). The antisense probe for Dlx 5 corresponds to an EcoRI to $A v a$ I fragment that contains the homeobox, which can distinguish the Dlx5 and $\delta D l x 5$ transcripts on the basis of size (see Fig. 1A). We used an antisense probe for the rpL32 ribosomal protein as a control for RNA loading (Shen and Leder, 1992). Quantitation of relative transcript abundances was performed by phosphorimager analysis and was normalized to the $r p L 32$ control.

RT-PCR analysis was performed by using total RNA from 6.5 through 11.5 dpc embryo fragments prepared with the RNeasy Mini kit (Qiagen, Hilden, Germany). The oligonucleotide primers for Dlx 5 flank the region deleted from $\delta D l x 5$ (see Fig. $1 A$ ) and are 5'-GGC CGC CGC AGT AGA AGA ACA-3' and 5'-GTG GGC ATG AGG GTG GTG GCT GAG-3'. The sequences of the control glyceraldehyde phosphate dehydrogenase (GAPDH) primers are 5'-GGC CAT GTA GGC CAT GAG-3' and 5'-AAA GCT GTG GCG TGA TGG-3'. PCR amplification was performed for 35 cycles at an annealing temperature of $68^{\circ} \mathrm{C}$ for the Dlx 5 primers and $59^{\circ} \mathrm{C}$ for the GAPDH primers. PCR products were resolved by agarose gel electrophoresis and were visualized by ethidium bromide staining and Southern blotting.

In situ hybridization was performed on whole mounts or cryosections of mouse embryos at the indicated developmental stages, using digoxygenin-labeled riboprobes (Sciavolino et al., 1997; Shen et al., 1997). The antisense probe corresponded to the full-length Dlx5 cDNA; no staining was detected, using a sense probe as a negative control (data not shown). Note that the in situ procedure cannot distinguish between expression of $D l x 5$ and $\delta D l x 5$ because the internal deletion that differs between these transcripts is relatively small ( $86 \mathrm{bp})$.

\section{RESULTS}

\section{DIx5 encodes two transcripts, one of which lacks the homeodomain}

To study the expression of murine $D l x 5$ as well as the biochemical properties of its protein product, we isolated $D l x 5$ cDNA clones
A

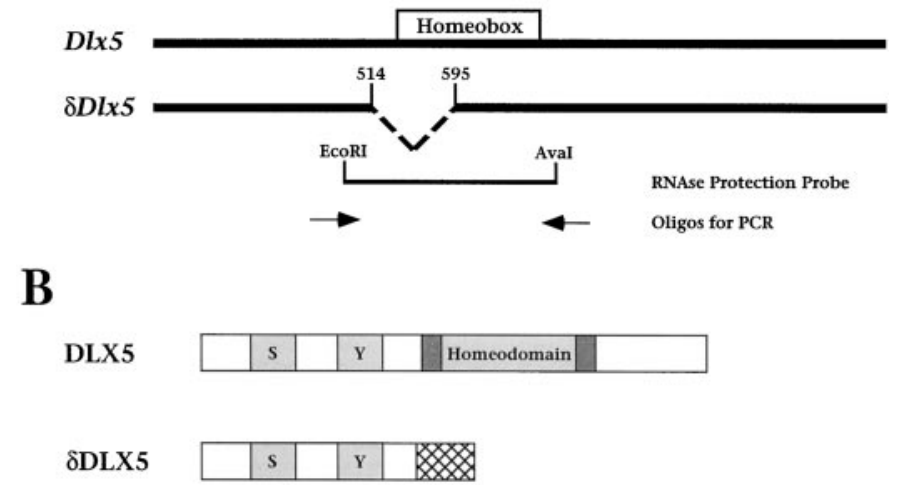

Figure 1. Dlx5 encodes two transcripts, one of which lacks an intact homeodomain. $A$, Schematic diagram depicting the Dlx5 and $\delta D l \times 5$ transcripts (GenBank accession numbers AF072452 and AF072453, respectively). Note that $\delta D l x 5$ represents an internal deletion of nucleotides 514-595 (inclusive) of the Dlx5 transcript [sequence numbering of Stock et al. (1996)]. Otherwise, $\delta D l \times 5$ is identical to the full-length transcript and contains the same $5^{\prime}$ and $3^{\prime}$ untranslated sequences. Shown are the positions of the ribonuclease protection probe (an EcoRI to AvaI restriction fragment) and the primers used for RT-PCR analysis. $B$, Diagram depicting the protein regions of DLX5 and 8 DLX5. Shown are the homeodomain and three regions conserved among DLX proteins, indicated by shaded boxes. Two of these conserved regions are serine-rich (indicated by $S$ ) and tyrosine-rich (indicated by $Y$ ), whereas the third comprises sequences directly flanking the homeodomain [the extended homeodomain (Zhang et al., 1997)]. Because of a frameshift, $\delta$ DLX5 contains the $\mathrm{N}$-terminal conserved domains, but lacks the homeodomain and other $\mathrm{C}$-terminal regions, and has a novel stretch of 28 amino acids (indicated by the cross-hatched box).

by screening a cDNA library derived from $12.5 \mathrm{dpc}$ embryonic head mRNA. Four of five cDNA clones isolated in this screen are full-length or nearly full-length ( $\sim 1400$ base pairs), in agreement with the published sequence (Stock et al., 1996). Conceptual translation of the coding region reveals three notable features of the DLX5 protein product: (1) a homeodomain that is flanked by conserved sequences [known as the extended homeodomain (Zhang et al., 1997)]; (2) a serine-rich region that contains putative phosphorylation sites, because DLX5 is phosphorylated in vitro (H. Zhang and C. Abate-Shen, unpublished data); and (3) an unusual tyrosine-rich motif, for which the function presently is unknown (Fig. 1A).

Notably, we found that one of our Dlx 5 cDNA isolates, which we call $\delta D l x 5$, contains an internal nucleotide deletion within the coding region but is otherwise identical in sequence to the other four cDNAs. This internal deletion creates a frameshift such that the predicted protein product ( $\delta$ DLX5) lacks the homeodomain and $\mathrm{C}$-terminal sequences but includes 28 novel amino acids (Fig. $1 B)$. These findings are in agreement with a recent study by Liu and colleagues (1997), in which they reported alternative transcripts for both $D l x 5$ and $D l x 6$, including one $(D l x-5 \gamma)$ that is similar to $\delta D l x 5$. Inspection of the nucleotide sequences adjacent to the internal deletion of $\delta D l \times 5$ reveals the presence of imperfect splice sites in the full-length cDNA (specifically a $5^{\prime}$-donor splice site at nucleotide 513 and a $3^{\prime}$-acceptor splice site at nucleotide 596; Fig. 1A). Therefore, this variant transcript may result from alternative splicing.

To verify that this $\delta D l x 5$ transcript is expressed in vivo and is not an artifact of cDNA synthesis, we performed ribonuclease protection analysis, using total RNA obtained from midgestation mouse embryos (Fig. $2 A$ ). For this analysis the riboprobe corre- 


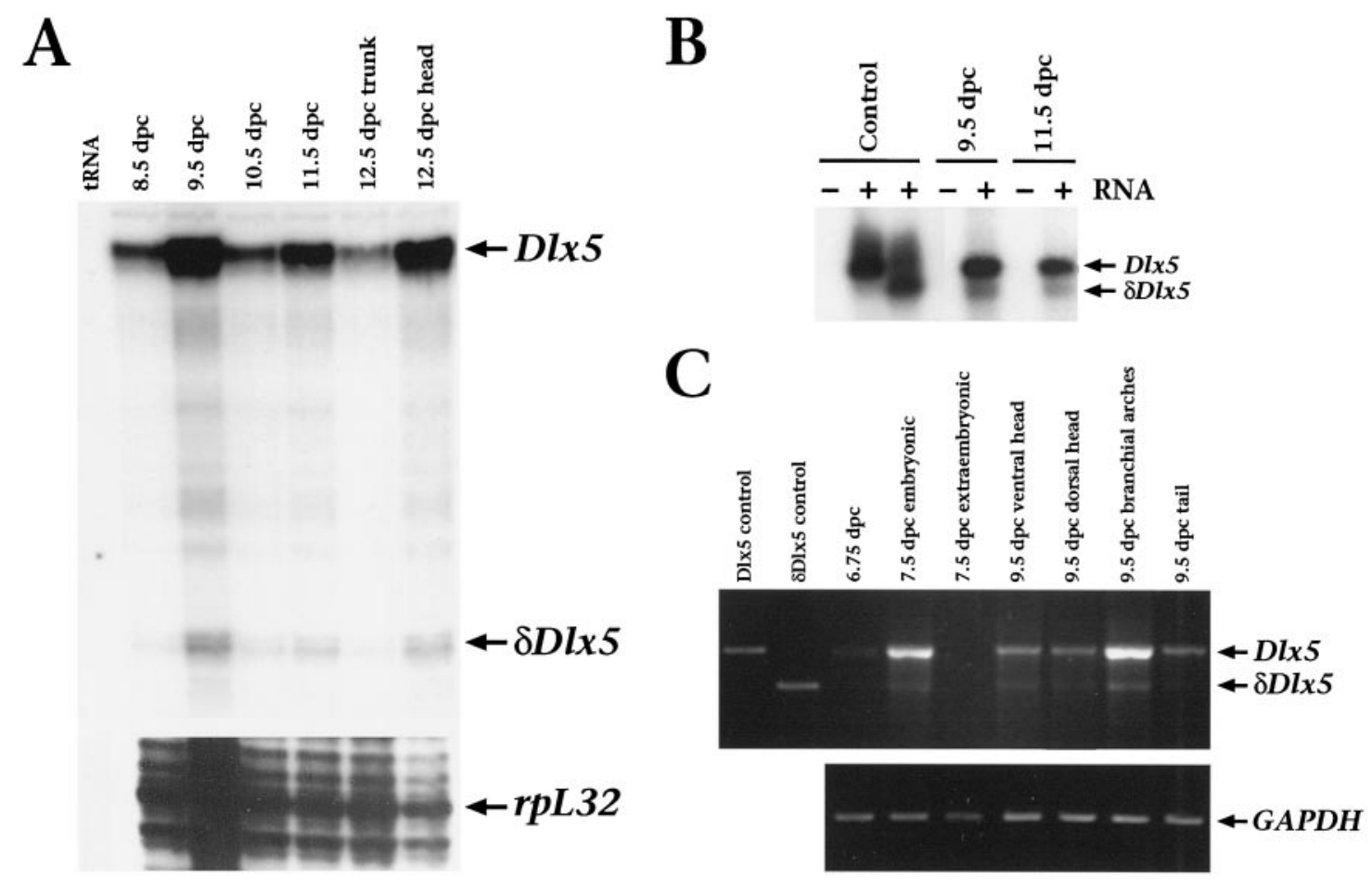

Figure 2. Detection of $D l x 5$ and $\delta D l x 5$ transcripts in mouse embryonic tissues. $A$, Ribonuclease protection analysis of $D l x 5$ and $\delta D l x 5$ expression was performed by using total RNA from whole mouse embryos or from torso or head at the developmental stages indicated. The antisense probe protects a fragment of 253 nucleotides for the Dlx5 transcript and a fragment of 171 nucleotides for the $\delta D l x 5$ transcript (see Fig. $1 A$ ). Arrows indicate the positions of the $D l x 5, \delta D l x 5$, and the $L 32$ (internal control) transcripts. No signal was observed with a control sense riboprobe (data not shown). $B$, RT-PCR analysis of Dlx5 and $\delta D l x 5$ transcripts was performed in the absence (-) or presence (+) of Dlx5 or $\delta D l x 5$ cDNA (Control) or total RNA prepared from 9.5 or $11.5 \mathrm{dpc}$ whole embryos. PCR products were subjected to Southern blot analysis and visualized by autoradiography. The oligonucleotides used for RT-PCR flank the region deleted in $\delta D l x 5$ such that the two products can be distinguished on the basis of their size (see Fig. $1 A$ ). $C$, RT-PCR analysis of Dlx5 and $\delta D l x 5$ transcripts was performed on Dlx5 or $\delta D l x 5$ cDNA controls or on total RNA extracted from dissected embryo fragments of the indicated stages. RT-PCR analysis of GAPDH was performed in parallel reactions as a positive control for cDNA synthesis. PCR products were resolved by agarose gel electrophoresis and visualized by ethidium bromide staining.

sponded to the homeobox region of Dlx5 such that the intact $(D l \times 5)$ and alternative $(\delta D l \times 5)$ transcripts can be distinguished on the basis of their size (see Fig. $1 A$ ). Although the overall abundance of $D l x 5$ transcripts varies among the developmental stages that were examined, both $D l x 5$ and $\delta D l x 5$ are detected at each stage (Fig. $2 A$ ). Moreover, quantitation of these results shows that the abundance of $\delta D l x 5$ represents $\sim 10 \%$ of the total Dlx5 transcripts in each of these RNA populations.

We further verified that $\delta D l x 5$ is expressed during development by performing RT-PCR analysis. For this purpose we designed oligonucleotide primers that distinguish the Dlx5 and $\delta D l \times 5$ transcripts on the basis of size (see Fig. $1 A$ ). As shown in Figure $2 B$, two distinct PCR products were detected in 9.5 and $11.5 \mathrm{dpc}$ total RNA isolated from embryonic heads. The upper band is of greater abundance and comigrates with the product obtained from the control Dl $x 5$ cDNA, whereas the lower band is less abundant and comigrates with the product obtained from the control $\delta D l x 5$ cDNA.

We used this RT-PCR assay to examine further the expression of Dlx5 and $\delta D l x 5$ transcripts in earlier developmental stages and tissues (Fig. 2C). We found that Dlx5 was barely detected in 6.75 dpc egg cylinders and was abundant in 7.5 dpc embryonic tissues, but not extraembryonic tissues, and also was detected readily in $9.5 \mathrm{dpc}$ ventral head, dorsal head, branchial arches, and tail (Fig. $2 C)$. $\delta D l \times 5$ was expressed in parallel with $D l \times 5$ at each of these stages but at lower abundances (Fig. 2C). Together with the results of the ribonuclease protection analysis, these findings demonstrate that $\delta D l \times 5$ is, indeed, expressed in vivo, representing $\sim 10 \%$ of the total Dlx5 transcripts throughout development.

\section{The $\delta \mathrm{DLX} 5$ protein does not interact with DNA or its protein partner MSX1}

Because the predicted $\delta$ DLX5 protein lacks a homeodomain, we anticipated that its biochemical properties might differ significantly from those of the full-length DLX5 protein. To test this prediction, we performed gel mobility shift assays, using DLX5 and $\delta$ DLX5 proteins that were obtained by in vitro transcription/ translation of the corresponding cDNAs. The DNA sites used for this analysis were variants of a homeodomain consensus DNA site, which contains an essential TAAT core and flanking nucleotides that contribute to DNA-binding specificity (Catron et al., 1993). As shown in Figure $3 A$, full-length DLX5 interacts with these DNA sites to varying degrees, although it does not bind to a DNA site containing a mutated TAAT core. In contrast, SDLX5 does not interact with any of the DNA sites that were tested, consistent with its lack of a homeodomain.

In previous studies we have shown that the biochemical properties of DLX proteins, including DLX5, are modulated via their interactions with the MSX1 homeoprotein and that the MSXDLX interaction is mediated by their respective homeodomains (Zhang et al., 1997). Therefore, we further compared the biochemical properties of DLX5 and 8 DLX 5 by examining their ability to form protein complexes with MSX1. As shown in Figure $3 B$, the full-length DLX5 protein interacts well with a GST- 
A

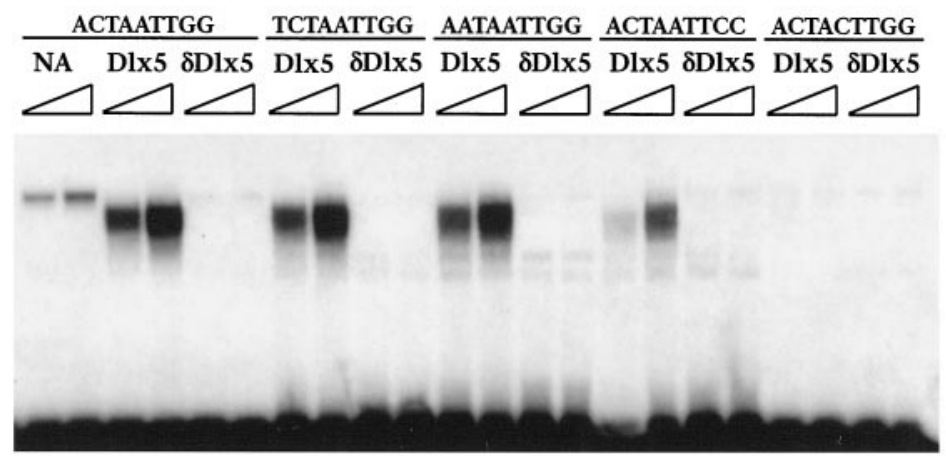

B

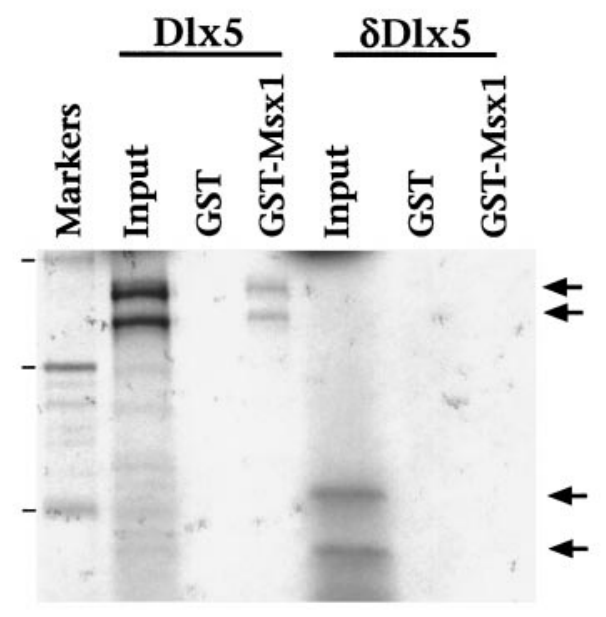

Figure 3. $\delta \mathrm{DLX} 5$ does not interact with DNA or exhibit homeodomain-dependent protein-protein interactions. $A$, Gel mobility shift analysis was performed as described (Zhang et al., 1997), using DLX5 or $\delta$ DLX 5 proteins obtained by in vitro transcription/translation $(1.0$ or $2.5 \mu \mathrm{l}$, as indicated by the triangle). Sequences of the DNA sites that were used are shown (Catron et al., 1993). The lane labeled $N A$ represents unprogrammed lysate. $B$, GST protein interaction assay was performed by using ${ }^{35}$ S-labeled DLX 5 or $\delta$ DLX 5 proteins $(5 \mu \mathrm{l})$ and a GST or GST-MSX1 fusion protein $(5 \mu \mathrm{g})$. The ${ }^{35} \mathrm{~S}$ labeled proteins were immobilized on GST resin and resolved by SDS-polyacrylamide gel electrophoresis, followed by autoradiography. The input lanes contain $20 \%(1 \mu \mathrm{l})$ of the total ${ }^{35} \mathrm{~S}$-labeled protein that was used in the GST interaction assay, and the marker lane shows the positions of ${ }^{14} \mathrm{C}$-labeled molecular size standards corresponding to 46, 31, and $19 \mathrm{kDa}$ (arrowheads).
MSX1 fusion protein, but not with GST alone, whereas $\delta$ DLX5 does not interact at all with GST-MSX1. Together with the results of the DNA binding analysis, these findings demonstrate that $\delta$ DLX5 is deficient in the biochemical activities that require the homeodomain.

\section{DIx5 expression marks the rostrolateral boundaries of the neural plate}

In the course of our analyses of expression patterns of Dlx family genes (Zhang et al., 1997), we observed that Dlx5 is expressed at much earlier stages of development than have been reported previously for any member of the murine Dlx family (Price et al., 1991; Robinson et al., 1991; Bulfone et al., 1993a; Simeone et al., 1994; Sheng et al., 1997). Therefore, we have examined Dlx5 expression by in situ hybridization during gastrulation and neurulation stages of mouse embryogenesis.

Notably, our findings demonstrate an early phase of Dlx5 expression that demarcates the rostrolateral boundaries of the prospective neuroectoderm. In particular, we have found that Dlx5 expression first can be detected at the late-streak stage of gastrulation $(6.75 \mathrm{dpc})$, which precedes the formation of an overt neural plate, but is not expressed at earlier stages (Figs. 4, 5; data not shown). (It is important to note that, because Dl $x 5$ and $\delta D l x 5$ differ only in a small region, the resolution of the in situ approach does not distinguish expression of these transcripts; thus we refer to the total signal detected as Dlx5 expression.) During early gastrulation, at $6.75 \mathrm{dpc}, \operatorname{Dl} x 5$ expression is detected in a graded rostrocaudal distribution as a lateral band on the embryonic side of the embryonic/extraembryonic junction (Fig. 4A,B). Sections from whole-mount embryos at this stage show that this stripe of Dlx5 expression is localized to the ectoderm layer and is not found in the overlying mesoderm or visceral extraembryonic endoderm (Fig. $5 A$ ). At early neural plate stages $(7.25 \mathrm{dpc}) \operatorname{Dl} x 5$ is expressed in a circumferential stripe around the embryonic half of the egg cylinder (see Fig. 4C,D). The rostral extent of this stripe overlaps the position at which the head folds will emerge, whereas the caudal end curls dorsally at the base of the allantois. Interestingly, the anterior limit of Dlx 5 expression at these stages is localized to the most rostral region of the ectoderm and abruptly terminates where it adjoins the amnion (Fig. 5B,C).

By the head-fold stages of gastrulation $(7.5 \mathrm{dpc}), \mathrm{D} l \times 5$ expression appears more intense in the rostral ectoderm of the emerging head folds, whereas its expression laterally and caudally is less intense (see Fig. $4 E-G$ ). At these stages the expression of $D l x 5$ clearly defines the emerging anterior neural ridge (Fig. $5 D-F$ ). After gastrulation, at early somite stages of development (8.0 dpc), Dlx5 expression continues to be restricted to the anterior head folds, whereas its expression laterally and caudally fades (Figs. $4 H, J, L, 5 H$ ). In addition, it is noteworthy that $D l x 5$ expression disappears from the rostral midline starting at this stage (see Fig. 4I,M, arrowheads). Thus, these findings demonstrate that $D l x 5$ is an early marker of the rostral ectoderm before the formation of the anterior neural ridge and that its subsequent expression specifically demarcates the anterior neural ridge.

In addition to marking the anterior neural ridge, Dl $x 5$ also is 

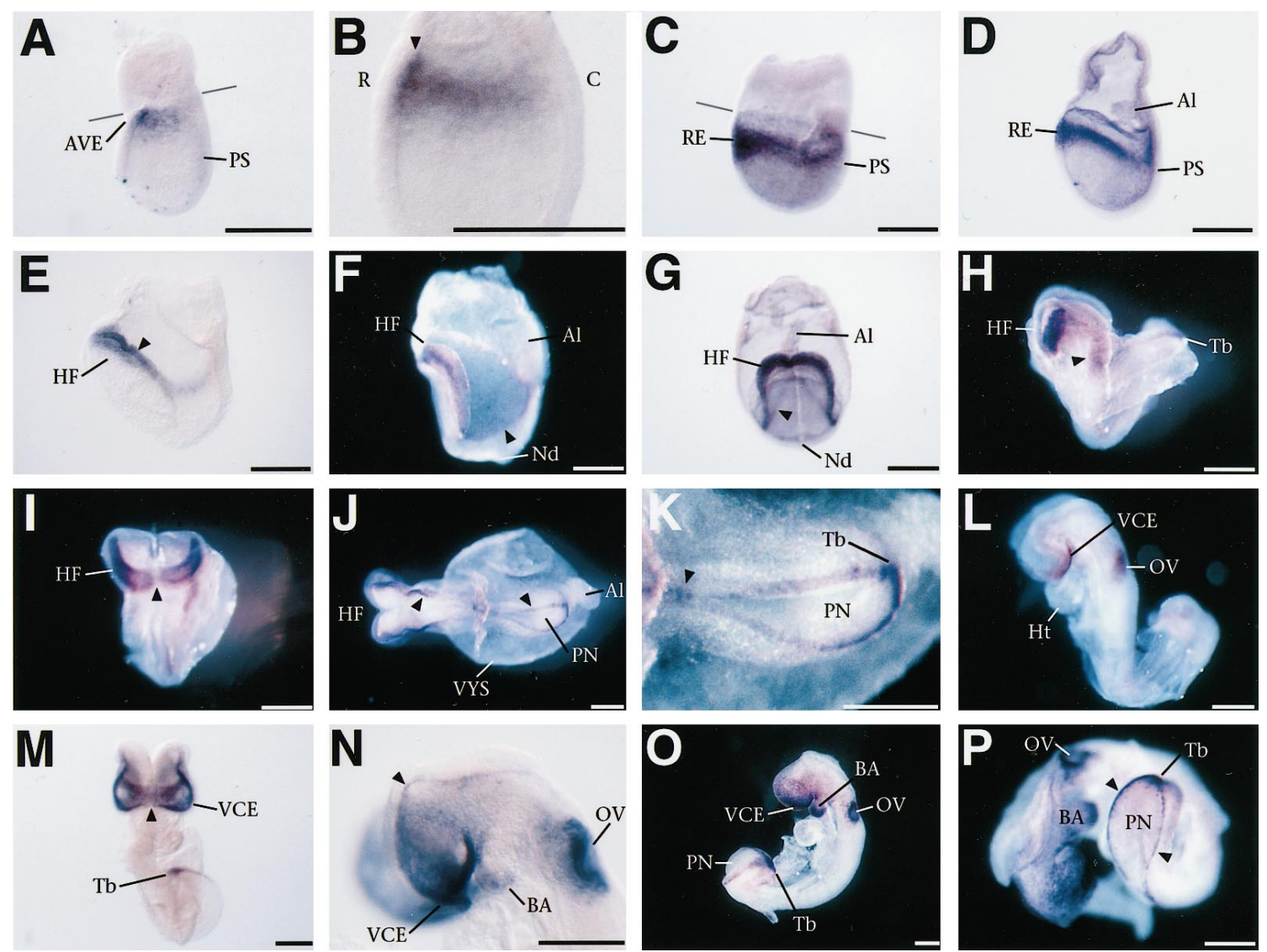

Figure 4. Expression of Dlx5 in whole-mount mouse embryos during gastrulation and neurulation stages. In all panels except $G, I$, and $M$, anterior (rostral) faces toward the left. A, Lateral bright-field view of a late-streak stage egg cylinder $(6.75 \mathrm{dpc})$, showing expression in a lateral band just below the embryonic/extraembryonic junction. The gray lines mark the boundary between the embryonic and extraembryonic halves of the egg cylinder. $B$, High-power view of an embryo similar to that in $A$, showing the rostrocaudal gradient of Dlx5 expression, with the highest level at the rostral end (arrowhead). C, Early neural plate stage embryo $(7.25 \mathrm{dpc})$, with expression in a broad circumferential band that is widest rostrally and curls dorsally at the base of the emerging allantois. $D$, Late neural plate stage $(7.5 \mathrm{dpc})$, showing more intense rostral expression, with the band of expression fainter and narrower caudally. $E$, Early head-fold stage $(7.75 \mathrm{dpc})$, showing expression at the lateral edges of the neural plate $($ arrowhead $) . F$, Lateral dark-field view at the late head-fold stage $(8.0 \mathrm{dpc})$, showing expression in a thin lateral stripe rostrally that is barely detectable caudally (arrowhead). $G$, Frontal bright-field view of an embryo similar to that shown in $F$. Expression is strongest at the anterior margin of the neural plate and diminishes caudolaterally (arrowhead). H, Lateral view of a two-somite embryo $(8.25 \mathrm{dpc})$, with strong expression detectable only in the ventral ectoderm beneath the head folds and with weak expression in the otic placode (arrowhead). I, Frontal view of the embryo shown in $H$. Note that rostral expression is weaker in the midline (arrowhead).J, Dorsal view of a four-somite embryo, showing expression at the lateral edges of the neural groove before neural tube closure (arrowheads). $K$, Higher-power view of the posterior neuropore of the embryo shown in $J$. Note that weak expression can be detected after neural tube closure (arrowhead). L, Lateral view of a six-somite embryo, showing expression in the ventral cephalic epithelium underneath the head folds and in the developing otic vesicles. $M$, Frontal view of an eight-somite embryo, showing bilateral expression in the ventral cephalic epithelium before anterior neuropore closure, but not at the midline (arrowhead). $N$, High-power lateral view of the embryo shown in $M$, showing staining in the ventral cephalic epithelium that continues faintly at the boundary of the anterior neuropore (arrowhead). Also shown is staining in the otic vesicle and in the newly forming mesenchyme of the first branchial arch. $O$, Lateral view of a 10 -somite embryo $(8.5 \mathrm{dpc})$, showing prominent expression in the ventral cephalic epithelium, the first branchial arch, the otic vesicles, and the tail bud. $P$, Higher-power view of an embryo similar in stage to that in $O$, showing staining in the tail bud and in the lateral edges of the posterior neuropore (arrowheads). Al, Allantois; $A N$, anterior neuropore; $A V E$, anterior visceral endoderm; $B A$, first branchial arch; $C$, caudal; $H F$, head folds; $H t$, heart; $N d$, node; $N T$, neural tube; $O V$, otic vesicle; $P N$, posterior neuropore; $P S$, primitive streak; $R$, rostral; $R E$, rostral ectoderm; $T b$, tail bud; $V C E$, ventral cephalic epithelium; $V Y S$, visceral yolk sac. Scale bars, $200 \mu \mathrm{m}$.

expressed at the lateral margins of the neural plate at late stages of gastrulation $(7.75 \mathrm{dpc}$ ) (see Fig. $4 F, G)$. During early somite stages $(8.25 \mathrm{dpc})$ this lateral zone of Dlx5 expression is found in a narrow strip of cells adjacent to the neuroepithelium along the length of the neural plate, marking the position of the presumptive premigratory neural crest (Figs. $4 J, K, P, 5 M, N$ ). Unlike the rostral zone of expression, the lateral zone of $D l \times 5$ expression disappears shortly after neural tube closure (see Fig. 4O). Thus, the lateral domain of $D l x 5$ expression may correspond to the premigratory neural crest.

Before anterior neuropore closure $(8.0 \mathrm{dpc})$ the rostral zone of
Dlx5 expression becomes restricted to the ventral cephalic epithelium and olfactory placodes, whereas expression is not found in the prosencephalon (see Fig. $4 L-N$ ). Sections from this stage clearly demonstrate that $D l \times 5$ expression is restricted to the non-neural cephalic epithelium and is excluded from the overlying neural tissue (Fig. 5H-J). After anterior neuropore closure (8.5 dpc) Dlx5 expression continues in the ventral cephalic epithelium and also is found in the cranial neural crest derivatives that form the mesenchyme of the first branchial arch (Figs. 4O,P, $5 K, L)$. At $10.5 \mathrm{dpc}$, expression of $D l \times 5$ is evident in the olfactory epithelium of the nasal cavity (Fig. $5 Q$ ). This expression contin- 
ues in the olfactory epithelium through $13.5 \mathrm{dpc}$ and is also prominent in the vomeronasal (Jacobson's) organs, which are derived from the olfactory epithelium (Fig. $5 R-T$ ).

It is noteworthy that the expression of $\operatorname{Dlx} 5$ in the anterior neural ridge and its derivatives contrasts with a distinct domain of its expression in the telencephalon and diencephalon, which has been described previously (Simeone et al., 1994; Chen et al., 1996; Sheng et al., 1997) and which resembles the expression pattern of several other Dlx genes (Porteus et al., 1991, 1994; Price et al., 1991; Robinson et al., 1991; Bulfone et al., 1993a,b; Simeone et al., 1994). Notably, Dlx5 expression is not detected in the prosencephalon at $8.5 \mathrm{dpc}$ (Fig. $5 I, K)$, whereas beginning at $9.5 \mathrm{dpc} D l \times 5$ expression is observed in two separate regions of the ventral telencephalon and diencephalon (Fig. 5O,P).

\section{DISCUSSION}

In this report we describe two aspects of $D l x 5$ expression that may offer insight into its function during neurogenesis. First, we show that a significant percentage of $D l x 5$ transcripts encodes a protein ( $\delta$ DLX5) that lacks a homeodomain and presumably would have altered biological functions in vivo. Second, our in situ hybridization studies define an early phase of $D l \times 5$ expression at the rostral and lateral borders of the neural plate. Notably, the expression of Dlx5 at the rostral boundary of the neural plate and in the anterior neural ridge occurs at the developmental stages when these regions have organizing activities that pattern the adjacent rostral prosencephalon (Shimamura and Rubenstein, 1997; Houart et al., 1998). These observations underscore the complex regulation and potential activities of members of the $D l x$ family.

\section{Potential for regulation of DLX5 activity by an alternative transcript}

An unexpected finding of our study was the identification of a second Dlx5 transcript, $\delta D l \times 5$, which encodes a truncated polypeptide that lacks biochemical activities requiring the homeodomain. Interestingly, in their analysis of Dlx5 expression in later developmental stages, Rubenstein and colleagues also have reported recently the isolation of two alternative $D l \times 5$ transcripts, one of which $(D l x-5 \gamma)$ corresponds to the transcript we call $\delta D l x 5$ (Liu et al., 1997). Here, we demonstrate that $\delta D l \times 5$ is deficient in several biochemical activities and is coexpressed with the fulllength transcript in similar ratios at early developmental stages.

We can envisage several possible roles for truncated versions of the DLX5 protein. First, $\delta$ DLX 5 may be inactive in all functional respects, in addition to those that we have examined. In this regard, the production of an alternative transcript may provide a means of regulating the levels of active DLX5 protein in vivo. Second, $\delta$ DLX5 may be active in some functional contexts, but not in others. If so, $\delta$ DLX 5 may provide a means of regulating DLX5 activity in a dominant-negative manner via competition with DLX5 for components of transcription complexes. Indeed, $\delta$ DLX5 contains the N-terminal regions required for transcriptional activation, which are conserved among DLX proteins (G.
$\mathrm{Hu}$ and C. Abate-Shen, unpublished data); thus $\delta$ DLX5 could compete with full-length DLX5 for interactions with other transcription factors. Third, $\delta$ DLX 5 may have distinct biochemical activities that do not require the homeodomain. Notably, Ftz polypeptides lacking the homeodomain are nonetheless biologically active during Drosophila embryogenesis (Copeland et al., 1996). Furthermore, numerous homeobox genes, including HoxA1, HoxB6, CASP, and CSX1/Nkx2.5, have alternative transcripts that encode truncated proteins lacking the homeodomain (Baron et al., 1987; LaRosa and Gudas, 1988; Shen et al., 1991; Shiojima et al., 1996; Lievens et al., 1997). Although the functions of these truncated proteins have not yet been elucidated, the prevalent occurrence of alternative transcripts among homeoboxcontaining genes suggests that such transcripts have biological significance.

\section{Expression of DIx5 defines the anterior neural ridge}

The molecular processes by which the rostral ectoderm is patterned are of particular interest because this region gives rise to both neural and non-neural derivatives. Thus, the fates of the rostral ectoderm have been elucidated primarily by chick-quail transplantation studies (Couly and Le Douarin, 1985, 1987), which have been supported by DiI labeling experiments in cultured mouse embryos (Osumi-Yamashita et al., 1994). These fate-mapping studies suggest that the murine anterior neural ridge subsequently will generate ectodermal derivatives (adenohypophysis, ventral cephalic epithelium, and olfactory placodes), whereas regions that are caudal to the anterior neural ridge will generate structures of the forebrain (optic vesicles, hypothalamus, and ventral telencephalon) (Fig. 6). The anterior neural ridge extends caudolaterally and is contiguous with the lateral margins of the neural plate, which will generate the cranial neural crest (Couly and Le Douarin, 1985, 1987; Baker and Bronner-Fraser, 1997).

Our description of Dlx5 expression in the mouse embryo is in good agreement with the avian fate map (Fig. 6), because murine Dlx5 is expressed first in the anterior neural ridge and subsequently in the ectoderm of the nasal cavity, olfactory placodes, and ventral cephalic epithelium. Indeed, murine Dlx5 expression persists in these derivatives of the anterior neural ridge, whereas the disappearance of $D l \times 5$ expression in the rostral midline at early somite stages is consistent with its later absence in Rathke's pouch (adenohypophysis). It is important to note that the later phase of Dlx5 expression in ventral telencephalon and diencephalon occurs in regions that, based on the avian fate map, are not derived from the anterior neural ridge (Fig. 6) (Couly and Le Douarin, 1985, 1987), suggesting that these two phases of murine Dlx5 expression are distinct both in their temporal onset and embryological origin.

Among other molecular markers for regions of the anterior ectoderm in the mouse embryo are the homeobox genes Otx2 (Simeone et al., 1992, 1993; Ang et al., 1994), Pax6 (Li et al., 1994), and Six3 (Oliver et al., 1995) as well as the winged helix

\footnotetext{
epithelium of the nasal cavity and the ventral telencephalon. $R, S$, Coronal sections through a $13.5 \mathrm{dpc}$ embryo. Prominent expression of $D l x 5$ is observed in the olfactory epithelium and vomeronasal (Jacobson's) organs as well as in the developing cartilage of the nasal septum, dental epithelium of the developing teeth, ciliary ganglion, and the craniofacial mesenchyme. $4 V$, Fourth ventricle; $A l$, allantois; $A m$, amnion; $A N R$, anterior neural ridge; $A V E$, anterior visceral endoderm; $B A$, first branchial arch; $C$, caudal; $C F M$, craniofacial mesenchyme; $C G$, ciliary ganglion; $C M$, cephalic mesenchyme; $D E$, dental epithelium; $D i$, diencephalon; $E$, ectoderm; $F g$, foregut pocket; $H b$, hindbrain; $H F$, head folds; $H t$, heart; $M$, mesoderm; $N G$, neural groove; $N S$, nasal septum; $O E$, olfactory epithelium; $O P V$, optic vesicle; $O V$, otic vesicle; $P C M$, precardiac mesoderm (anterior lateral plate mesoderm); $P r$, prosencephalon; $P S$, primitive streak; $R$, rostral; $R E$, rostral ectoderm; $S o$, somite; $T e$, telencephalon; $T o$, tongue; $T V$, telencephalic vesicle; $V C E$, ventral cephalic epithelium; $V M O$, vomeronasal (Jacobson's) organ; $V Y S$, visceral yolk sac. Scale bars, $50 \mu \mathrm{m}$.
} 

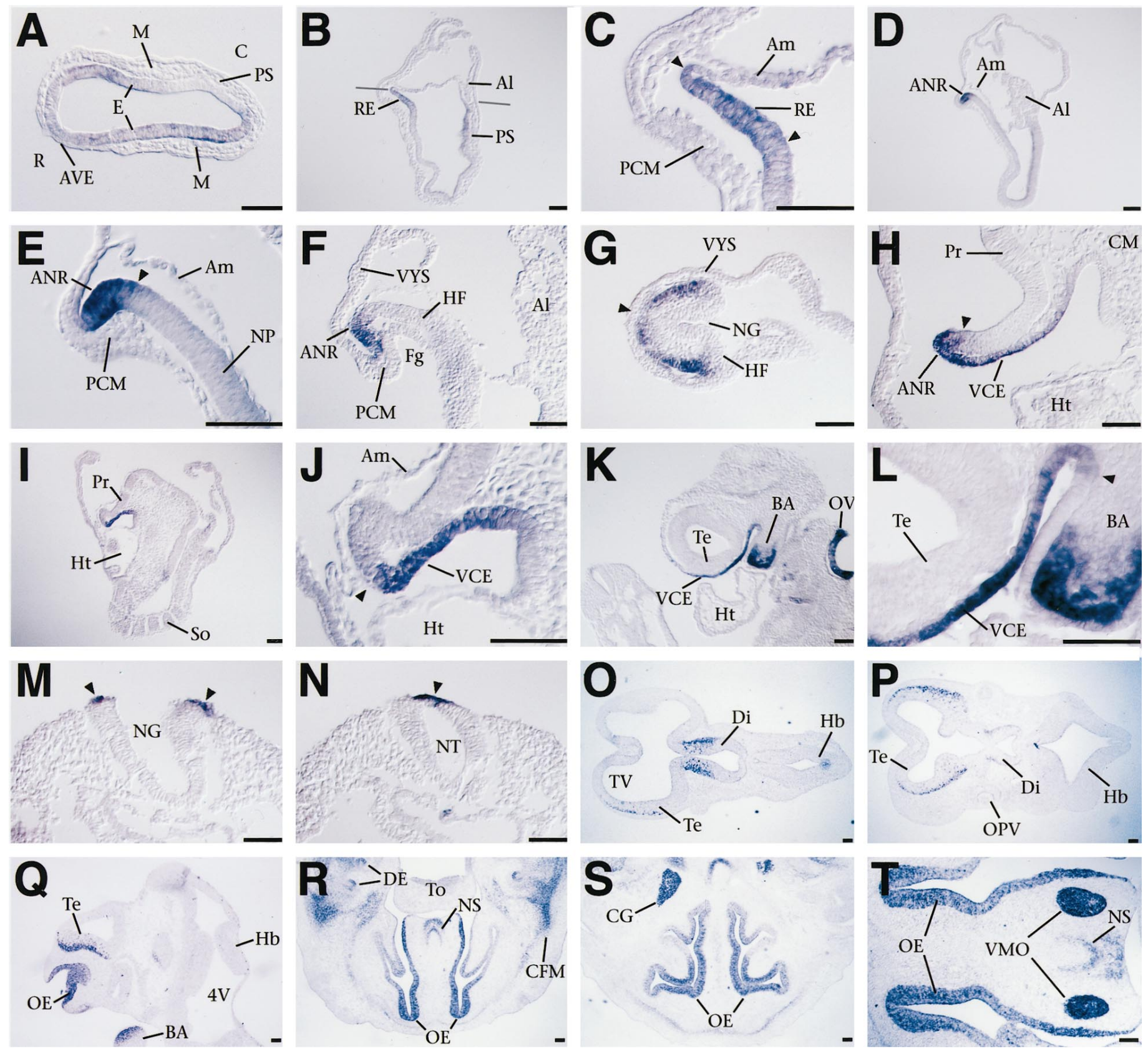

Figure 5. Expression of Dlx5 in mouse embryo sections. $A-N$ represent cryosections of embryos processed for whole-mount in situ hybridization, whereas $O-T$ show results obtained from section in situ hybridization. Anterior (rostral) faces to the left, except for $M, N, R$, and $S$, where anterior faces down. A, Transverse section through a late-streak stage embryo $(6.75 \mathrm{dpc})$, showing expression in the rostral and lateral ectoderm. Expression is not detected in the caudal ectoderm, near the primitive streak, nor in the newly formed mesoderm or in the extraembryonic visceral endoderm. $B$, $C$, Sagittal section through an early neural plate stage embryo $(7.25 \mathrm{dpc})$, showing expression in the rostral ectoderm as well as in the posterior ectoderm overlying the primitive streak. (The tissue layers have separated slightly during processing for cryosectioning.) The gray lines mark the boundary between the embryonic and extraembryonic regions of the egg cylinder. In $C$, note that expression in the rostral ectoderm overlies the precardiac mesoderm (anterior lateral plate mesoderm), with a sharp boundary rostrally at the junction with the extraembryonic amnion and a more diffuse boundary caudally (arrowheads). D, E, Sagittal view of an early head-fold stage embryo $(7.75 \mathrm{dpc})$, showing the formation of the anterior neural ridge. Again, note the relatively diffuse caudal boundary of expression (arrowhead). $F$, Sagittal section of a two-somite stage embryo, showing restricted expression of $D l x 5$ in the anterior neural ridge. $G$, Transverse section through the head folds at the two-somite stage. Expression is found in the ventral epithelium of the head folds, but expression does not extend into the neural groove and is nearly undetectable at the ventral midline (arrowhead). H, Higher-power sagittal view of a four-somite embryo, closer to the midline. Expression of Dlx 5 extends through the anterior neural ridge but terminates at the border with the prosencephalon (arrowhead). I, J, Sagittal section of a six-somite embryo. Note that expression is not detected in any region of the brain at this stage. $K, L$, Sagittal section through an $8.5 \mathrm{dpc}$ embryo, with expression in the ventral cephalic epithelium, mesenchyme of the first branchial arch, and otic vesicle. In $L$, note that expression of Dlx 5 in the ventral cephalic epithelium terminates caudally at the junction with the epithelium of the first branchial arch (arrowhead). $M, N$, Transverse sections through an $8.5 \mathrm{dpc}$ embryo at different axial levels. In the open neural groove of the posterior neuropore $(M)$, expression is found in cells adjacent to the columnar neuroepithelium (arrowheads). More rostrally, after neural tube closure $(N)$ transient expression is found in the cells overlying the dorsal neural tube (arrowhead). $O, P$, Coronal sections through a $9.5 \mathrm{dpc}$ embryo, showing the earliest detectable expression of $D l x 5$ in the forebrain. Expression primarily localizes to differentiating cells in the subventricular zone, although limited staining can be observed in the ventricular layer of the diencephalon. $Q$, Sagittal section through the head of a 10.5 dpc embryo, with expression in the olfactory 


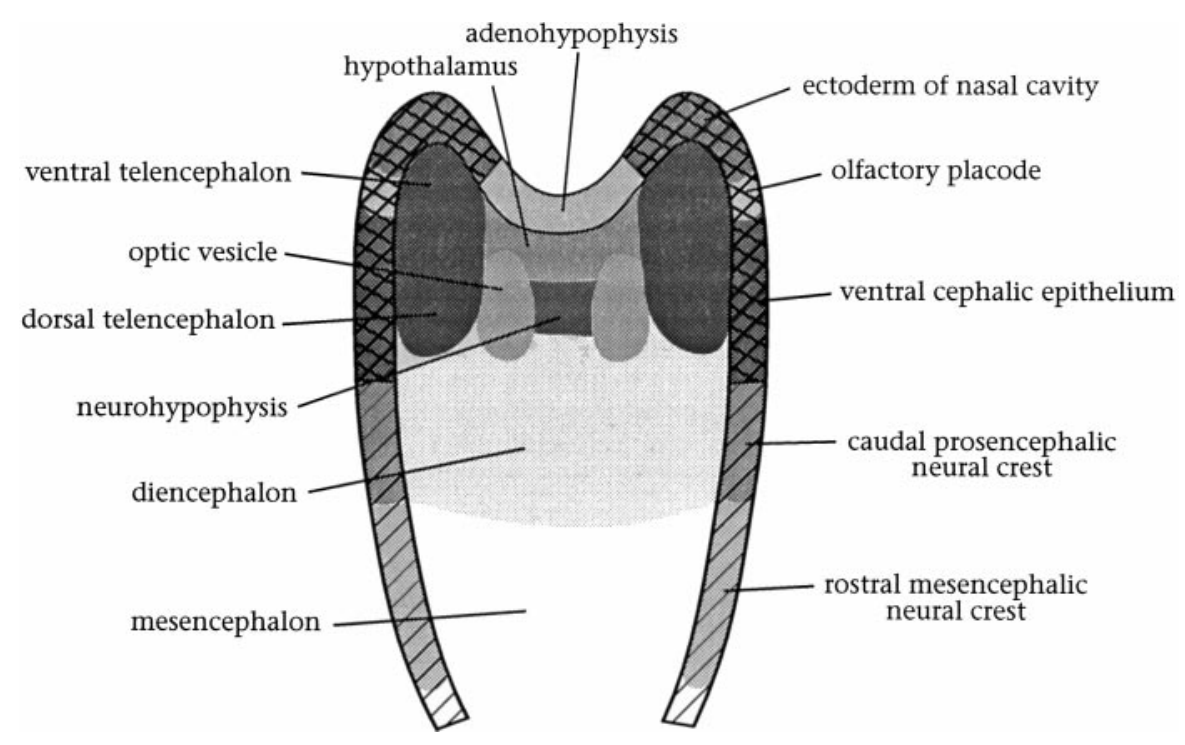

Figure 6. Fate map of the anterior ectoderm in the avian embryo, superimposed with the expression pattern of murine $D l x 5$. The fate map of the avian ectoderm layer at the three- to four-somite stage is redrawn from Couly and Le Douarin (1987). Cross-hatching denotes regions of higherlevel Dlx 5 expression at this stage, whereas lighter striping denotes lower-level expression (based on data in Figs. 4, 5). transcription factor BF1 (Tao and Lai, 1992). At early stages of neurulation these genes are expressed in overlapping patterns that include the anterior neural ridge and extend caudally to the forebrain (Oliver et al., 1995; Shimamura and Rubenstein, 1997). Subsequently, Six3, Otx2, Pax6, and BF1 are expressed continuously in specific domains of the forebrain (e.g., optic vesicles, hypothalamus, and ventral telencephalon) (Tao and Lai, 1992; Simeone et al., 1993; Li et al., 1994; Oliver et al., 1995). In contrast, $D l \times 5$ is not expressed in any forebrain regions until 9.5 dpc. This comparison suggests that regional subdivisions of the rostral ectoderm already may be established at the time of the formation of an overt neural plate.

\section{Expression of Dlx5 in the anterior neural ridge may be conserved evolutionarily}

Recent phylogenetic analyses of gene sequences have clarified the ortholog relationships among members of the vertebrate Dlx family, which has been complicated by a confusing nomenclature. In particular, the murine $D l x 5$ gene is orthologous to rat $r D l x$, chicken $g D l x 5$, Xenopus $X$-Dll3, and zebrafish $z D l x 4$ (Ferrari et al., 1995; Stock et al., 1996). Of these orthologous genes, the expression patterns of $X$-Dll3 and $z D l x 4$ have been examined during gastrulation and neurulation. In Xenopus, $X$-Dll3 is expressed during the neural plate stage along the anterior transverse ridge of the neuroectoderm, which is analogous to the murine anterior neural ridge, and in older embryos is expressed in the olfactory placodes, part of the cement gland, and in clusters of cells in the prosencephalon (Papalopulu and Kintner, 1993) in a pattern resembling that of murine Dlx5. In zebrafish, however, $z D l \times 4$ is expressed in a pattern similar to that of murine $D l \times 5$ at late stages of neurulation, but it is not expressed during gastrulation (Akimenko et al., 1994), suggesting that another (as yet uncharacterized) member of the zebrafish $D l x$ family may mimic the early expression pattern of murine $D l \times 5$.

Our findings demonstrate that Dlx5 is expressed at much earlier stages than has been reported previously for other members of the murine Dlx family. Interestingly, the single Distal-less homolog in Amphioxus, AmphiDll, is expressed during gastrulation by ectodermal cells at the rostrolateral margins of the neural plate and by presumptive neural crest cells (Holland et al., 1996). Holland and colleagues have proposed that AmphiDll represents an ancestral chordate $D l x$ gene, subsuming all of the functions of vertebrate $D l x$ genes, and that these functions have been split among different members of the Dlx family during evolution (Holland et al., 1996). This proposal is consistent with the unique early expression of murine $D l x 5$ in the anterior neural ridge and, in combination with its evolutionary conservation of expression, suggests a role in early neural patterning.

\section{REFERENCES}

Akimenko MA, Ekker M, Wegner J, Lin W, Westerfield M (1994) Combinatorial expression of three zebrafish genes related to Distal-less: part of a homeobox gene code for the head. J Neurosci 14:3475-3486.

Anderson SA, Eisenstat DD, Shi L, Rubenstein JLR (1997a) Interneuron migration from basal forebrain to neocortex: dependence on $D l x$ genes. Science 278:474-476.

Anderson SA, Qiu M, Bulfone A, Eisenstat DD, Meneses J, Pedersen R, Rubenstein JL (1997b) Mutations of the homeobox genes Dlx-1 and Dlx-2 disrupt the striatal subventricular zone and differentiation of late born striatal neurons. Neuron 19:27-37.

Ang S-L, Conlon RA, Jin O, Rossant J (1994) Positive and negative signals from mesoderm regulate the expression of mouse Otx2 in ectoderm explants. Development 120:2979-2989.

Baker CVH, Bronner-Fraser M (1997) The origins of the neural crest. Pt I, Embryonic induction. Mech Dev 69:3-11.

Baron A, Featherstone MS, Hill RE, Hall A, Galliot B, Duboule D (1987) Hox-1.6: a mouse homeo-box-containing gene member of the Hox-1 complex. EMBO J 6:2977-2986.

Bulfone A, Kim HJ, Puelles L, Porteus MH, Grippo JF, Rubenstein JLR (1993a) The mouse Dlx-2 (Tes-1) gene is expressed in spatially restricted domains of the forebrain, face, and limbs in midgestation mouse embryos. Mech Dev 40:129-140.

Bulfone A, Puelles L, Porteus MH, Frohman MA, Martin GR, Rubenstein JLR (1993b) Spatially restricted expression of $D l x-1, D l x-2$ (Tes1), Gbx-2, and Wnt-3 in the embryonic day 12.5 mouse forebrain defines potential transverse and longitudinal segmental boundaries. J Neurosci 13:3155-3172.

Catron KM, Iler N, Abate C (1993) Nucleotides flanking a conserved TAAT core dictate the DNA binding specificity of three murine homeodomain proteins. Mol Cell Biol 13:2354-2365.

Chen X, Li X, Wang W, Lufkin T (1996) Dlx5 and Dlx6: an evolutionary conserved pair of murine homeobox genes expressed in the embryonic skeleton. Ann NY Acad Sci 785:38-47.

Copeland JWR, Nasladka A, Dietrich BH, Krause HM (1996) Patterning of the Drosophila embryo by a homeodomain-deleted Ftz polypeptide. Nature 379:162-165.

Couly GF, Le Douarin NM (1985) Mapping of the early neural primordium in quail chick chimeras. I. Developmental relationships between placodes, facial ectoderm, and prosencephalon. Dev Biol 110:422-439.

Couly GF, Le Douarin NM (1987) Mapping of the early neural primordium in quail chick chimeras. II. The prosencephalic neural plate and 
neural folds: implications for the genesis of cephalic human congenital abnormalities. Dev Biol 120:198-214.

Ferrari D, Sumoy L, Gannon J, Sun H, Brown AM, Upholt WB, Kosher RA (1995) The expression pattern of the Distal-less homeoboxcontaining gene $D l x-5$ in the developing chick limb bud suggests its involvement in apical ectodermal ridge activity, pattern formation, and cartilage differentiation. Mech Dev 52:257-264.

Holland ND, Panganiban G, Henyey EL, Holland LZ (1996) Sequence and developmental expression of AmphiDll, an Amphioxus Distal-less gene transcribed in the ectoderm, epidermis, and nervous system: insights into evolution of craniate forebrain and neural crest. Development 122:2911-2920.

Houart C, Westerfield M, Wilson SW (1998) A small population of anterior cells patterns the forebrain during zebrafish gastrulation. Nature 391:788-792.

LaRosa GJ, Gudas LJ (1988) Early retinoic acid-induced F9 teratocarcinoma stem cell gene ERA-1: alternate splicing creates transcripts for a homeobox-containing protein and one lacking the homeobox. Mol Cell Biol 8:3906-3917.

Li HS, Yang JM, Jacobson RD, Pasko D, Sundin O (1994) Pax-6 is first expressed in a region of ectoderm anterior to the early neural plate: implications for stepwise determination of the lens. Dev Biol 162:181-194.

Lievens PM, Tufarelli C, Donady JJ, Stagg A, Neufeld EJ (1997) CASP, a novel, highly conserved alternative-splicing product of the CDP/cut/ cux gene, lacks cut-repeat and homeo DNA-binding domains, and interacts with full-length CASP in vitro. Gene 197:73-81.

Liu JK, Ghattas I, Liu S, Chen S, Rubenstein JLR (1997) Dlx genes encode DNA-binding proteins that are expressed in an overlapping and sequential pattern during basal ganglia differentiation. Dev Dyn 210:498-512.

Oliver G, Mailhos A, Wehr R, Copeland NG, Jenkins NA, Gruss P (1995) Six3, a murine homologue of the sine oculis gene, demarcates the most anterior border of the developing neural plate and is expressed during eye development. Development 121:4045-4055.

Osumi-Yamashita N, Ninomiya Y, Doi H, Eto K (1994) The contribution of both forebrain and midbrain crest cells to the mesenchyme in the frontonasal mass of mouse embryos. Dev Biol 164:409-419.

Papalopulu N, Kintner C (1993) Xenopus Distal-less related homeobox genes are expressed in the developing forebrain and are induced by planar signals. Development 117:961-975.

Porteus MH, Bulfone A, Ciaranello RD, Rubenstein JLR (1991) Isolation and characterization of a novel cDNA clone encoding a homeodomain that is developmentally regulated in the ventral forebrain. Neuron 7:221-229.

Porteus MH, Bulfone A, Liu JK, Puelles L, Lo LC, Rubenstein JLR (1994) DLX-2, MASH-1, and MAP-2 expression and bromodeoxyuridine incorporation define molecularly distinct cell populations in the embryonic mouse forebrain. J Neurosci 14:6370-6383.

Price M, Lemaistre M, Pischetola M, Di Lauro R, Duboule D (1991) A mouse gene related to Distal-less shows a restricted expression in the developing forebrain. Nature 351:748-750.

Puelles L, Rubenstein JLR (1993) Expression patterns of homeobox and other putative regulatory genes in the embryonic mouse forebrain suggest a neuromeric organization. Trends Neurosci 16:472-479.

Qiu M, Bulfone A, Martinez S, Meneses JJ, Shimamura K, Pedersen RA, Rubenstein JLR (1995) Null mutation of $D l x-2$ results in abnormal morphogenesis of proximal first and second branchial arch derivatives and abnormal differentiation in the forebrain. Genes Dev 9:2523-2538.
Robinson GW, Wray S, Mahon KA (1991) Spatially restricted expression of a member of a new family of murine Distal-less homeobox genes in the developing forebrain. New Biol 3:1183-1194.

Rubenstein JLR, Martinez S, Shimamura K, Puelles L (1994) The embryonic vertebrate forebrain: the prosomeric model. Science 266:578-580.

Sciavolino PJ, Abrams EW, Yang L, Austenberg LP, Shen MM, AbateShen C (1997) Tissue-specific expression of murine Nkx3.1 in the male urogenital system. Dev Dyn 209:127-138.

Shen MM, Leder P (1992) Leukemia inhibitory factor is expressed by the preimplantation uterus and selectively blocks primitive ectoderm formation in vitro. Proc Natl Acad Sci USA 89:8240-8244.

Shen MM, Wang H, Leder P (1997) A differential display strategy identifies Cryptic, a novel EGF-related gene expressed in the axial and lateral mesoderm during mouse gastrulation. Development 124:429-442.

Shen W-F, Detmer K, Simonitch-Eason TA, Lawrence HJ, Largman C (1991) Alternative splicing of the HOX 2.2 homeobox gene in human hematopoietic cells and murine embryonic and adult tissues. Nucleic Acids Res 19:539-545.

Sheng HZ, Bertuzzi S, Chiang C, Shawlot W, Taira M, Dawid I, Westphal $\mathrm{H}$ (1997) Expression of murine Lhx5 suggests a role in specifying the forebrain. Dev Dyn 208:266-277.

Shimamura K, Rubenstein JLR (1997) Inductive interactions direct early regionalization of the mouse forebrain. Development 124:2709-2718.

Shimamura K, Hartigan DJ, Martinez S, Puelles L, Rubenstein JLR (1995) Longitudinal organization of the anterior neural plate and neural tube. Development 121:3923-3933.

Shiojima I, Komuro I, Mizuno T, Aikawa R, Akazawa H, Oka T, Yamazaki T, Yazaki Y (1996) Molecular cloning and characterization of human cardiac homeobox gene CSX1. Circ Res 79:920-929.

Shirasawa T, Sakamoto K, Takahashi H (1994) Molecular cloning and evolutional analysis of a mammalian homologue of the Distal-less 3 (Dlx-3) homeobox gene. FEBS Lett 351:380-384.

Simeone A, Acampora D, Gulisano M, Stornaiuolo A, Boncinelli E (1992) Nested expression domains of four homeobox genes in developing rostral brain. Nature 358:687-690.

Simeone A, Acampora D, Mallamaci A, Stornaiuolo A, D'Apice MR, Nigro V, Boncinelli E (1993) A vertebrate gene related to orthodenticle contains a homeodomain of the bicoid class and demarcates anterior neuroectoderm in the gastrulating mouse embryo. EMBO J 12:2735-2747.

Simeone A, Acampora D, Pannese M, D'Esposito M, Stornaiuolo A, Gulisano M, Mallamaci A, Kastury K, Druck T, Huebner K, Boncinelli E (1994) Cloning and characterization of two members of the vertebrate Dlx gene family. Proc Natl Acad Sci USA 91:2250-2254.

Stock DW, Ellies DL, Zhao Z, Ekker M, Ruddle FH, Weiss KM (1996) The evolution of the vertebrate Dlx gene family. Proc Natl Acad Sci USA 93:10858-10863.

Tao W, Lai E (1992) Telencephalon-restricted expression of BF-1, a new member of the HNF-3/fork head gene family, in the developing rat brain. Neuron 8:957-966.

Wilson PA, Hemmati-Brivanlou A (1997) Vertebrate neural induction: inducers, inhibitors, and a new synthesis. Neuron 18:699-710.

Zhang H, Hu G, Wang H, Sciavolino P, Iler N, Shen MM, Abate-Shen C (1997) Heterodimerization of MSX and DLX homeoproteins results in functional antagonism. Mol Cell Biol 17:2920-2932. 